J.M. GRÉSILLON, E. SAUQUET, B. RENARD, M. LANG, E. LEBLOIS

Cemagref Lyon,

Unité de Recherche Hydrologie-Hydraulique 3 bis qual Chauveau, CP 220

69336 Lyon Cedex 09 gresillon@lyon.cemagref.fr

\title{
Changement climatique et événements extrêmes : crues, inondations, sécheresses. Que peut-on dire aujourd'hui ?
}

Le changement climatique rendra-t-il les événements hydrologiques extrêmes plus fréquents ? A partir d'une sélection de tests de détection de tendances dans les séries et d'une analyse critigue sévère de deux cents chroniques de debits journaliers, on montre qu'aucun changement généralisé à l'échelle de la France ne peut être décelé au cours du siécle dernier. Toutefois. quelques tendances cohérentes paraissent émerger au niveau régional. Ainsi, dans le Nord-Est de la France une legère aggravation des crues se dessinerait ; au contraire les étiages dans les Pyrénées semblent devenir plus sévères alors qu'ils le seraient moins dans les Alpes. Si les débits extrẻmes ne semblent pas avoir encore enregistré de modifications, les modèles de circulation gênérale (GCM) en revanche annoncent des évolutions futures conséquentes des températures et, dans une moindre mesure, des précipitations. Le bilan entre précipitation et évaporation sera neltement modifié et les modèles hydrologiques prévoient pour l'avenir des étiages beaucoup plus sévères, notamment au Sud de la France. Décrire précisément l'évolution des extrêmes à partir des GCMs est prématuré, le couplage des modèles hydrologiques et des modèles climatiques étant à ce jour difficile. Cependant, la prudence suggere de ne pas négliger ces éventuelles évohutions, au prétexte que des outils et données fiables pour les prévoír ne sont pas encore disponibles.

Mots-ciés : crues, sécheresses, non stationnarité, changement climatique, étude d'impact, modèle de circulation générale (GCM). France.

\section{Climate Change and Extreme Hydrological Events: What do we know today?} paper presents an analysis of stationarity performed on an extended data set of time series from wo hundred French gauging stations. This study involves the cautious selection of statistical tests to be carried out and a meticulous quality control of data to avoid drawing biased conclusions. Results do not provide any evidence of a generalized and consistent change at the scale of France during the last century. However, some consistent trends emerge at the regional scale: a weak increase of floods is detected in the North-East of France. whereas droughts seem more severe in the Pyrenean area and less extreme in the Alps. However Global Circulation Models (GCMs) forecast major evolutions of temperatures and signilicant, alhough contrasued, evolutions of rainfali. The balance between rainfall and evapotranspiration will therefore be strongly affected. Hydrologic rainfali-runoff models predict more severe droughts in French rivers, mainiy in the South of France. A more accurate assessment of climate change impacts on hydrological extremes is unfortunately impossible, notably 
because of scale inconsistencies between GCM and hydrological models. However cautiousness suggests that such evolutions cannot be disregarded even if available numerical tools are unable to calculate reliable numerical values.

Key words: floods, droughts, non-stationarity, climate change, impact study, global circulation model (GCM), France

menée dans le cadre d’un projet de recherche national (Lang et al., 2003; Renard et al., 2006 ; Lang et Renard, 2007). La seconde s'appuie sur des modèles climatiques pour regarder vers le futur. A partir de divers scénarios, elle analyse les impacts possibles du changement climatique sur le régime des rivères (Sauquet et al., 2005). en 1992 (41 morts), celles du Nord de la France en 1993, 1994 (21 morts) et 1995, puis celle de l'Aude en 1999 (29 morts) et celle du Gard en septembre 2002 (24 morts) ont amené les pouvoirs publics et la population à s'interroger sur l'origine de ces catastrophes. Des événements exceptionnels ont également touché l'Europe centrale et les Balkans avec les crues de l'Elbe, de la Vlava et du Danube (1997, 2002, 2005 et 2006). L'impack des activités anthropiques à l'échelle du bassin versant (types de cultures, aménagement de l'espace, urbanisation et imperméabilisation des sols, etc.] et, plus récemmenl, à une échelle globale (réchauffement de la planètej ont été invoqués. Au préalable, une analyse de l'évolution des régimes hydrologiques est requise pour identifier si, de manière objective, une dérive dans les données est perceptible. Une revue de la littérature montre que contrairement aux variables de temperature, aucune conclusion sur les écoulements ne peut être vallde à I'échelle mondiale. En Europe, Hisdal et al. (2001) ne décèlent aucune évolution dans les étiages sur plus de 600 stations. Plus localement, des modifications significatives et cohérentes peuvent apparaître. Une diminution des écoulements moyens et des étiages due à une réduction des précipitations est ainsi signalée en Turquie (Cigizoglu et al,. 2005) ; au Canada, une diminution des écoulements de fin de printemps due à une fonte des neiges devenue plus précoce est également notée (Zhang et al. 2001) alors qu'aux Etats-Unis, plusieurs analyses Indiquent une tendance récente vers des étages moins sévères sur une zone centrale comprise entre la frontière mexicaine et la région des Grands Lacs (Lins et Slack, 1999; Douglas et al., 2000: Groisman et al, 2005)

II semble tout aussi difficile de conclure quant à une aggravation généralisée des crues (Kundzewicz ef al., 2005). A ce jour, nous ne pouvons que soupçonner des changements dans les réponses hydrologiques et la rigueur scientifique impose d'en vérifier la présence dans un cadre méthodologique préalablement consolidé et đe mener, en complément, des études d'impact pour a imaginer $\$$ l'hydrologie future

Dans cet article, nous présentons deux types d'approches pour évaluer l'impact du changement climatig̣ue sur le régime des écoulements en France. notamment dans leurs extrêmes (crues et étiages). La première approche constitue un regard sur le passé (approche dite de détection) : à partir de données recueillies pendant au moins quarante ans sur un grand nombre de bassins versants français, elle rend compte d'une analyse statistique sur la détection des changements hydrologiques sur les rivières françaises

\section{Détection de changements hydrolo- giques à l'échelle de la France}

\section{1}

\section{Variabilité naturelle des régimes hydrologiques}

La détection de changements dans les régimes bydrologiques est une opération difficile. Elle consiste à identifier d'éventuelles modifications dans des signaux qui connaissent une variabilité temporelle intrinsèque : les rivières réagissent à des forçages complexes très variables (pluie, neige, température) qui eux-mêmes subissent des amortíssements ou, au contraire, des amplifications au travers de leurs bassins versants. Le comportement d'un bassin versant est donc difificile à caractériser et l'évolution future des débits à son exutoire est encore plus délicate à définir. La figure 1 représentant les variations des débits pendant quatorze années du Serein, mesuré à Chablis, fait apparaître presque chaque année une ou plusieurs valeurs fortes de débits. Le traitement statistique adéquat de ces valeurs fortes constitue un enjeu classique pour les hydrologues. En effet, la valeur des quantiles de période de retour influence les choix et dimensionnements des aménagements dans les vallées.

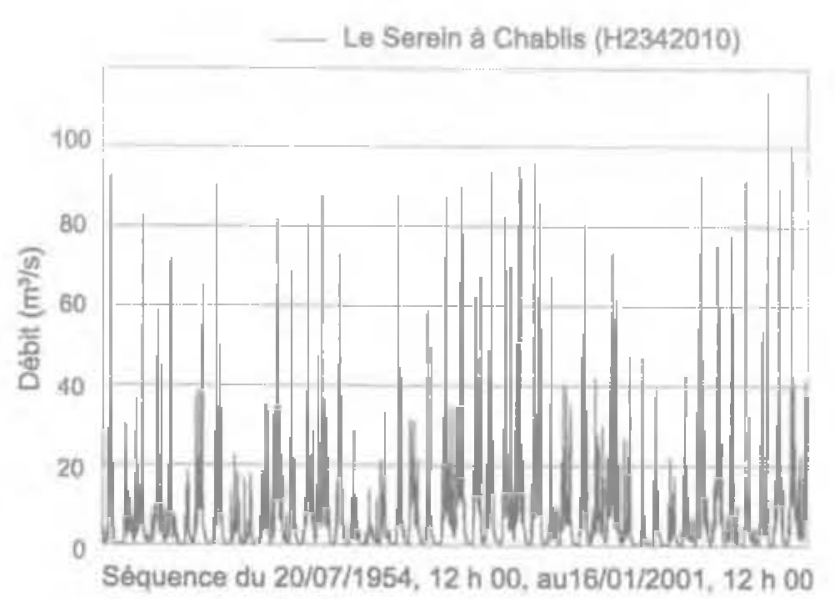

FG. 1 Variabilité “ naturelle $»$ des régimes hydrologiques (le Serein à Chablis).

Present climate variability in river flow time series (Serein River at Chablis). 
La précision des résultats du traitement statistique (et donc de l'évaluation des probabilités des événements rares) dépend notamment de la iongueur des séries observées ; il est, par exemple, difficile de définir la probabilité d'une valeur rare sur une courte série car elle s'appuie sur la partie extrapolée de la distribution statistique. Les séries d'observations sur les rivières françaises couvrant en général des périodes de quelques décades, la précision est en général faible ou très faible sur les quantiles de crue ayant une probabilité inférieure à 1/100 d’être dépassés chaque année (donc de a période de retour $) 100$ ans ou plus). Dans ces conditions, l'occurrence d'une valeur très forte est difficilement caractérisable : il peut s'agir d'un événement rare, relevé par hasard dans la période d'observation (problème d"échantillonnage). L'attribution d'une telle valeur à une a non stationnarité » du clima: n'est pas possible sans une démarche spécifique qui montrerait qu'il existe une évolution cohérente dans le temps et identifiable.

\section{2}

\section{Analyse locale et critique des données}

Une thèse récente (Renard, 2006) a recherché les éventuelles évolutions temporelles dans les séries hydrométriques. Près de deux cents stations ont foumi des chroniques de débits journaliers sur des périodes de quarante années ou davantage. Ces stations, gérées par les directions régionales de l'Environnement (DIREN], par EDF ou par la CNR sont réparties sur l'ensemble du territoire français, avec une concentration plus forte sur la région Centre et le Sud-Est de la France (Fig. 2). Selon les gestionnaires, ces stations sont censées fournir des données de bonne qualité et dénuées d’influences anthropiques.

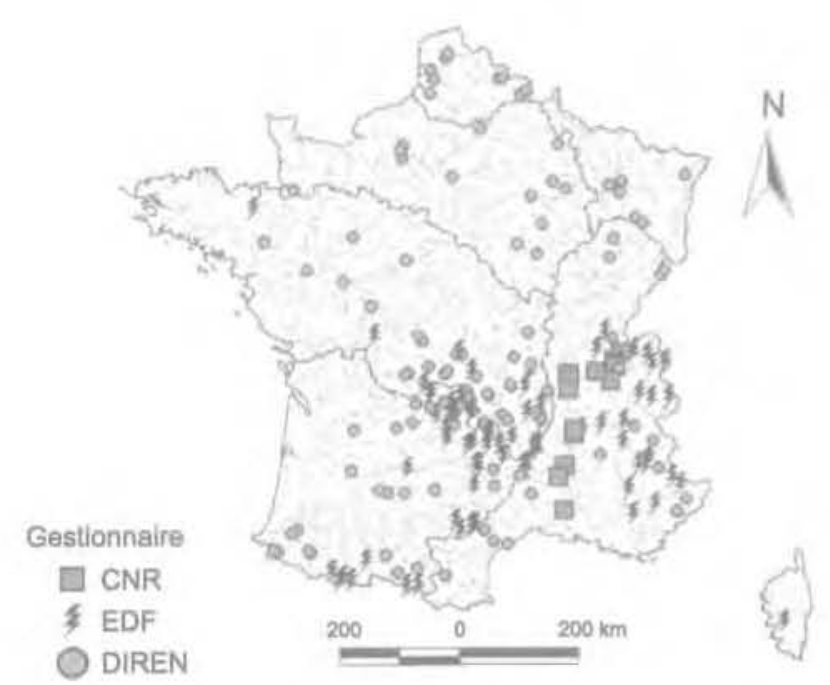

F6. 2 Localisation des stations de mesures des débits joumaliers exploitées dans l'analyse de stationnarité.

Gauging stations used in the stationarity analysis.

Différents types de changements dans les séries sont possibles : ruptures ou évolutions progressives indiquant des modifications dans les séries en moyenne ou variance. D'autre part, les variables à étudier peuvent décrire les hautes eaux, les basses eaux, les formes d'hydrogramme, etc. Pour chaque combi- raison de types de changement et de variable, un test d'identification a été choísi dans un ensemble de tests paramétriques et non paramétriques, selon une procédure systématique et préalablement contrôlée.

Les résultats de lanalyse font apparaître des changements relativement nombreux, aussi bien sur les variables de hautes eaux que sur celles descriptives des basses eaux. Par exemple, les séries de débits maxima annuels (MAXAN) sont considérées, selon ces tests, comme présentant des changements significatifs au seuil de $10 \%$ dans $27 \%$ des stations étudiées. Cependant, à l'échelle du territoire, il existe des hausses et des baisses (en proportion équivalente). Il ne semble pas exister de cohérence spatiale nette dans ces évolutions annoncées par les tests (Fig. 3). Au vu de ces résultats, une rế-analyse des données a été effectuée, avec un nouvel examen de l'historique de chacune des stations. Cette opération a condut à identifier, dans une proportion importante de stations, des interventions et travaux divers sur le dispositif de mesure susceptibles d'expliquer des différences dans les extrapolations des courbes de tarage et done des artefacts (rupture ou tendance d'origine purement métrologiquel dans les résultats des tests. Un tiers environ des stations a ainsi été écarté. L'analyse a été ensuite renouvelée sur l'échantillon réduit aux stations n'ayant pas subi ce type de perturbations.

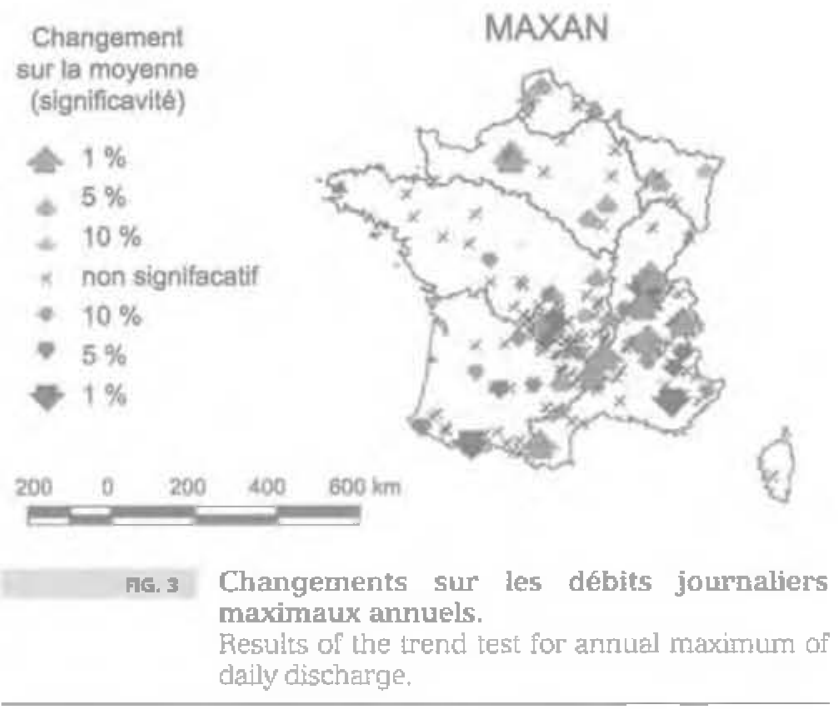

La proportion des séries composant l'échantillon réduít de stations affectées par des changements significatifs est deux fois plus faible que précédemment. Ces changements affectent le régime de crue (débits maximaux annuels, nombre d'événements de crues раг an, date) et même les étiages (durée, débit minimal moyen annuei sur 7 jours, date de ce minimum). Un exemple relatif aux crues est fourni sur la figure 4. On observe par exemple que les maxima annuels présentent beaucoup moins de changements significatifs qu'auparavant, qu'il existe encore des hausses et des baisses et que la cohérence spatiale des évolutions n'est toujours pas très grande. En ce qui concerne les étiages, les résultats sont similaires (peu de changements significatifs et absence de structure spatiale). L'analyse de ces résultats monte qu'aucune des variables (crue et étiagel n'a d'évolution significative à l'échelle de la France. Autrement dit, le nombre de changements détectés n'est pas suffisant pour rejeter l'hypothèse qu'ils soient tous dus au hasard. 


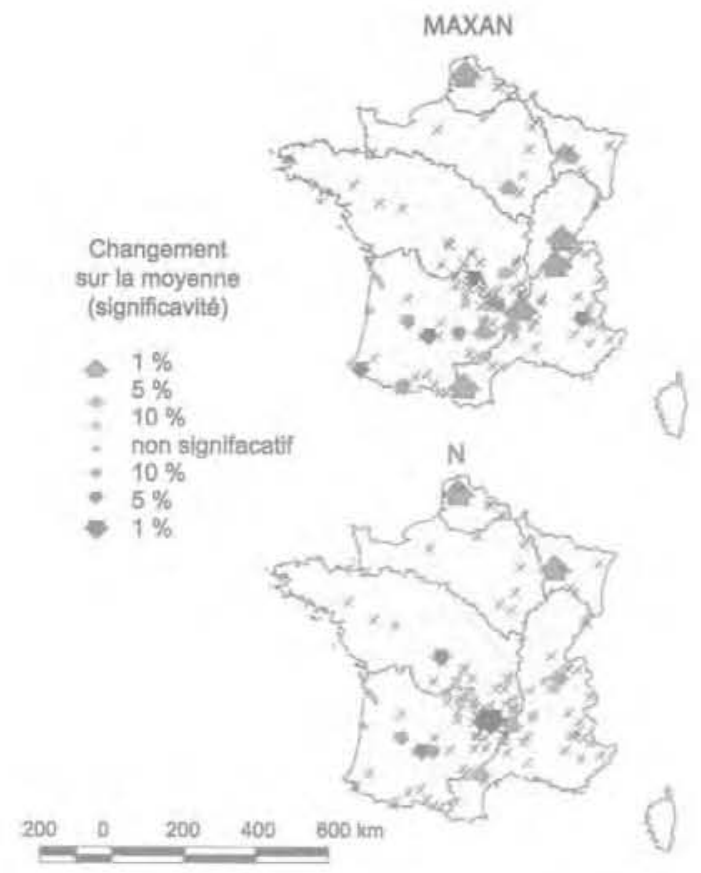

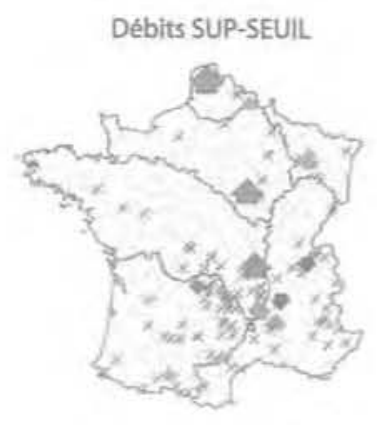

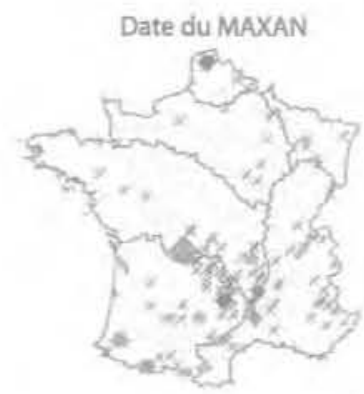

ตG. Ëvolutions relatives aux crues après critique des donnêes. maxan = maxima annuels des débits journaliers ; débit sup-seuil = pointe de débíts journaliers au dessus d'un seuil ; N = nombre moyen annuel d'evénements au-dessus d'un seuil ; date du Maxan = date du max annuel. Fesults of the trend tests after data analysis.

maxan = annual maxima of daily discharges; débil sup-seuil = maximum daily discharges above a fixed threshold; $\mathrm{N}=$ mean annual number of events above a fixed threshold; Date du Maxan = date of annual max.

\section{3}

\section{Analyse régionale pour la détection de changements hydrologiques}

L'absence de cohérence spatiale dans les résultats des études menées station par station a conduit à une analyse fondée sur des regroupements régionaux. Les stations hydrométriques ont été rassemblées en ensembles homogènes en croisant deux classifications, l'une fondée sur les pluies (Champeaux et Tamburini, 1995), l'autre sur le régime hydrologique (Pardé, 1955). Un test régional a été développé et appliqué pour analyser des évolutions consolidées sur ces regroupements. Peu de changements apparaissent ainsi, à trois exceptions près :

1) en région alpine, la sévérité des étiages diminue (la moyenne du débit mínimum sur 7 jours et celle du débit d'étiage sur 30 jours présentent une tendance significative à l'augmentation]. L'augmentation avérée des températures de l'air pourrait avoir réduit la quantité d'eau retenue en hiver sous forme de neige, augmentant ainsi les débits sur cette saison. De plus, les écoulements liés à la fonte des neiges semblent présenter une précocité accrue, et le volume écoulé entre juin et octobre semble augmenter pour les rivières soumises a une influence glaciaire. Ces éléments sont à mettre en relation avec les travaux sur le retrait des glaciers (Vincent, 2006) :

2) dans les Pyrénées au contraire, les étiages deviennent plus sévères. Dans une moindre mesure, l'assèchement est également sensible sur les écoulements annueis et sur les crues pluviales qui ont une tendance à la bajsse ;

3) dans le Nord-Est de la France apparaît une légère tendance à l'aggravation des crues décelée sur les séries de maxima annuels journaliers.
En conclusion, l'observation des régimes hydrologiques du passé ne permet pas, pour le moment, de mettre en évidence des changements d'ensemble très nets dans les séries temporelles. Pourtant, à l'échelle régionale, des changements cohérents apparaissent tout de même : augmentation des étiages dans les Alpes; diminution au contraire de ces étiages et de l'hydraulicité dans les Pyrénées ; légère augmentation des crues dans le Nord de la France.

Bien que ces êvolutions solent faibles et d'une amplitude telle qu'elles pourraient être interprétées comme de l'ordre des incertitudes, leur líaison avec l'évolution observée des températures et des précipitations les rend cohérentes (Renard, 2006). Il est nécessaire de surveiller ces évolutions qui pourraient s*amplifier dans les années à venir.

\section{3}

\section{Scénario climatique et hydrologie}

Tournant notre regard vers le futur, nous utiliserons les données des modèles de circulation générale pour tenter de prévoir quel climat ils proposent pour l'avenir et de traduire cette vision en terme d'hydrologie.

\section{1}

\section{Les modèles et leurs hypothèses}

Les modèles de circulation générale (GCM) reposent sur une description des processus physiques, chimiques et biologiques dans l'atmosphère, les océans, la cryosphère et sur les surfaces continentales à l'échelle de la planète. Iis résolvent les équations de la mécanique et de la thermodynamique des fluides géophysiques dans les trois dimensions spatiales et 
dans le temps sur les éléments d'un maillage recouvrant le globe. Ces éléments ont une résolution horizontale d'une centaine de kilomètres, et de quelques dizaines de mètres à un kilomètre sur l"axe vertical. Ils décrivent le climat passé et proposent une projection du climat futur en incluant notamment des évolutions de la composition en gaz à effet de serre. Les modèles diffèrent par leur résolution spatiale et par les lois physiques représentées. Ils ne sont malheureusement pas parfaits du fait de certains processus mal appréhendés à grande échelle. Ainsi, dans le cadre du projet GICC-Seine, Ducharne et al. (2004) soulignent un biais de reconstitution sur les précipitations mensuelles pouvant dépasser $50 \%$ sur le bassin de la Seine certains mois en hiver. Pour valoriser ces sorties, les hydrologues ont alors recours à une hypothèse forte et pourtant nécessaire : ils supposent que les défauts de reconstitution du modèle identifiés er reconstitution du passé seront présents et équivalents dans les chroniques du futur. En pratique, les sorties pluie et température sont de manière classique exploitées en terme d'u anomalies : (écarts) par rapport ał climat passé reconstitué par le GCM.

La suite exploite pour partie des scénarios SRES obtenus en 2001 et mis à disposition sur le site de l'IPCC (Intergouvemmental Panel on Clinate Change Data Distribution Centre http://ipcc-ddc.cru.uea.ac.uk/).

\section{2}

\section{Scénarios et anomalies saisonnières}

Le rôle du $\mathrm{CO}_{2}$ étant prépondérant dans le changement climatique, les scénarios envisagés reposent sur différentes hypothèses d'évolution de son contenu dans l'atmosphère. Les hypothèses sur les niveaux de croissance économique et de croissance démographique sont donc à la base des scénarios. L'un des plus pénalisants, mais réaliste, considère, à l'échelle de la planète, une croissance démographique qui se poursuit puis se stabilise au milieu du Xxie siècle, avec une croissance économique très rapide pourtant compensée par l'introduction de nouvelies technologies \& pro- pres » pour la production d’énergie (scénario A1). Avec ce scénario, à l'horizon 2080, le modèle CCSR/NIES AGCM prévoit, par rapport à la période 1960-1990, des élévations de température moyennes de juin à août qui se situent autour de quatre à cinq degrés sur la plupart des continents (Fig. 5). Il prévoit des anomałies de précipitations légèrement positives (accroissement) de décembre à février sur le nord des continents (Fig. 6) mais nettement négatives (diminution) en été au sud de l'hémisphère nord.

A une échelle spatiale plus fine, celle du bassin du Rhône (Fig. 7), un autre scénario correspondant à un doublement du $\mathrm{CO}_{2}$ à échéance de 2050, traité par deux autres modèles (modèle du CNRM de Météo France et modèle LMD Parisi) prévoit une augmentation des précipitations d'hiver et d'une diminution de celles-ci l'été ('Leblois et al., 2004).

\section{3}

\section{Impacts hydrologiques du changement climatique}

La taille des mailles des modèles de circulation générale et encore plus leur résolution temporelle sont beaucoup trop grandes pour permettre, directement, une approche fiable de l'impact du changement climatique sur les crues et les inondations. Tout juste peut-on prévoir que I'augmentation attendue des précipitations d"hiver pourrait induire une aggravation des crues et événements extrêmes.

En revanche les périodes d'étiage, provoquées par de longues périodes sans pluie et associées à des évaporations é évapotranspirations importantes, peuvent être étudiées à partir des sorties des GCMs. Sur plusieurs affluents du Rhône (la Drôme, le Roubion, I'Eyrieux), Sauquet et al. (2005) ont analysé les impacts prévisibles à l'échéance de 2050 du changement climatique sur les étiages, caractérisés par la variable QMNA5, débit mensuel minimal dans l'année, de période de retour 5 ans lou si on préfère, le débit mensuel minimal dans l'année qui aurait aujourd'hui une probabilité de

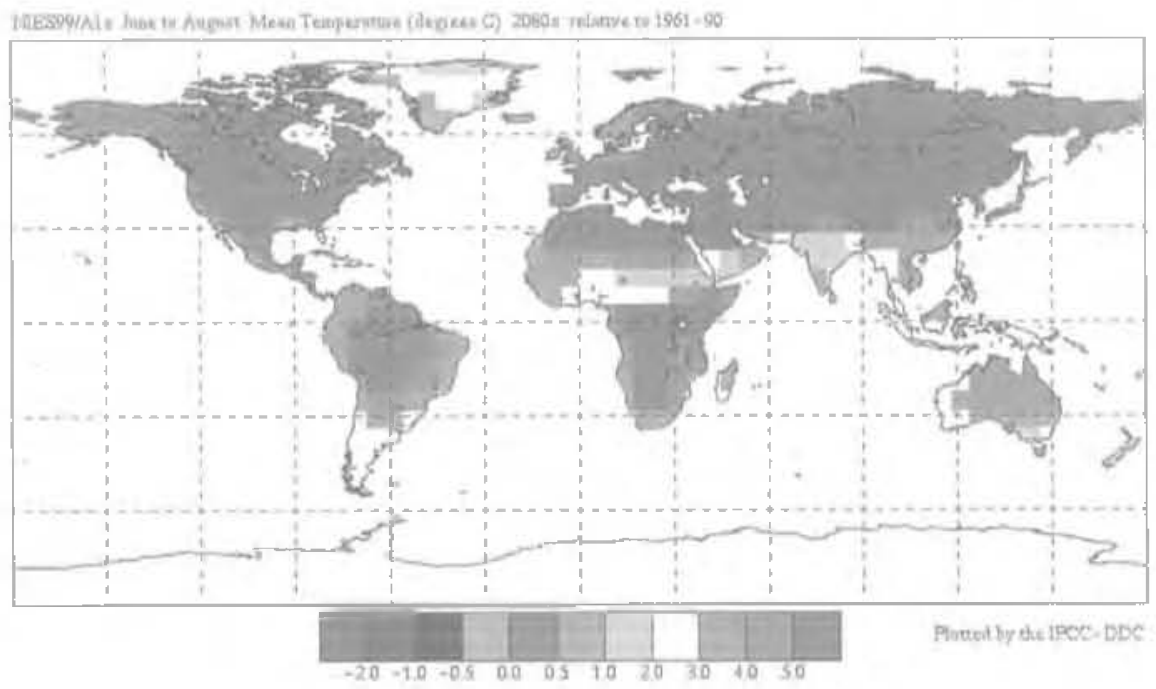

n3.s Anomalies de température à l'échéance 2080, pour la période juin-août. Modèle CCSR/NIES; scénario A1. Temperature anomalies at 2080 date; June to August period ; CCSRNIES model ; A1 scenario. 


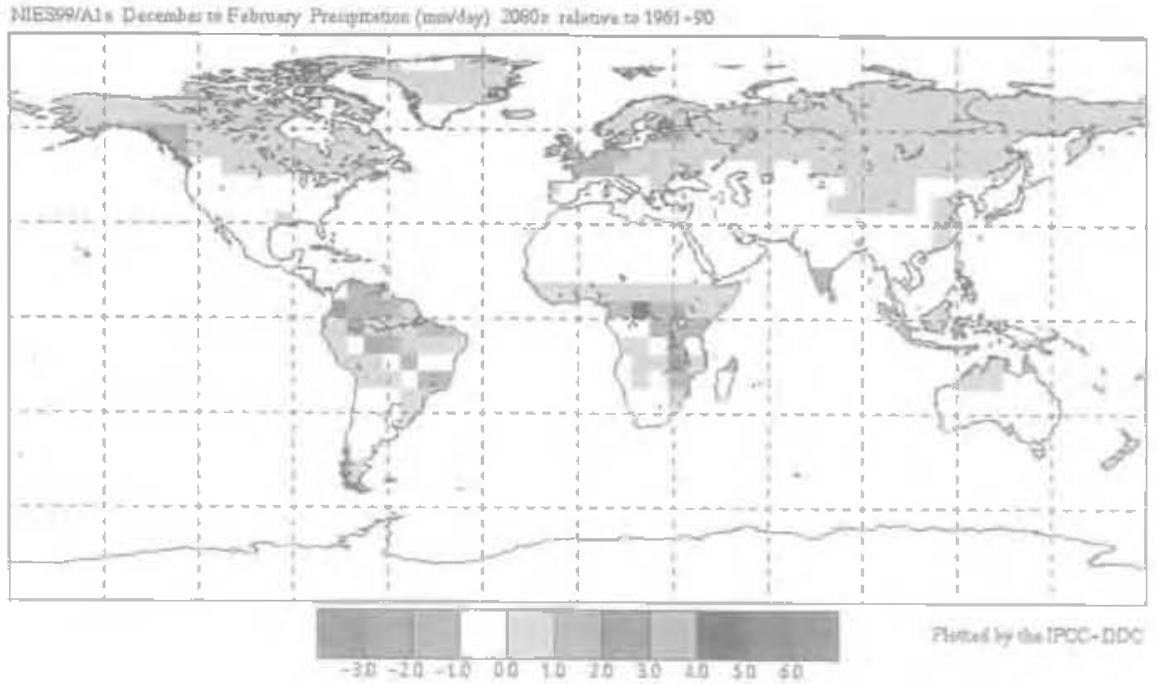

пlG. Anomaliesdeprécjpitationsàl'échéance2080, pourlapériodedécembre-février.ModèleCCSR/NIES ; scénarioA1 Rainfall anomalies at 2080 date ; Decenber to February pertod; CCSR/NIES mode]; A1 scenario.

$20 \%$ de ne pas être dépassé, ou une probabilité de $80 \%$ d'être dépasséł. Bien entendu, cette probabilité est jugée en référence à la période actuelle.
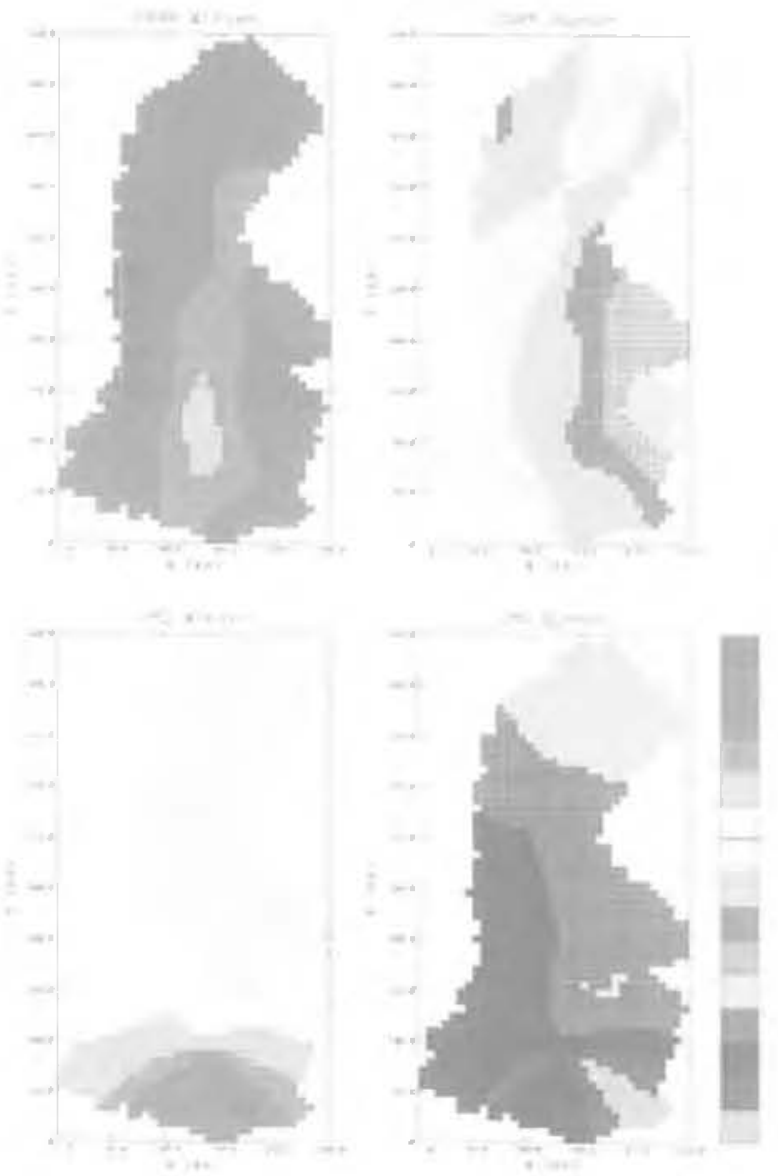

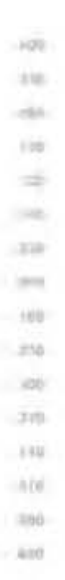

Figure 7 - Anomalies de précipitations sur le bassin du Rhône; scémario doublement de $\mathrm{CO}_{z}$ échéance 2050. Données LMD et MétéoFrance, mise en forme Météo-France.

Rainfall anomalies on the Rhone River basin at 2050 term; $\mathrm{CO}_{2}$ doubling scenario.
La méthode employée a consisté à caler, pour le climat présent, un modèle hydrologique et à l'appliquer pour le futur, en faisant l'hypothèse d'une transformation pluie débit stationnaire, et en affectant aux pluies actuelles la «perturbation n de grande échelle calculée par le GCM et en calcułant une évapotranspiration potentielle (ETP) tenant compte de l'élévation de température. Pour la Drôme, c'est le modèle conceptuel suédois Hydrologiska Byråns Vatterbalansavdelning HBV (Bergstrōm et Forsman, 1973 et décrit, par exemple, dans Perrin (2000)) qui donne les meilleures performances pour ka reconstitution du climat présent. La figure 8 représente les fonctions de répartition des valeurs des débits QMNA5 observés dans le climat présent (points noirs), celles des dẻbits du climat présent mais obtenus par simulation avec HBV évoqué ci-dessus (points rouges) et celles des débits simulés par ce même modèle sous hypothèses du changement climatique (points bleus).

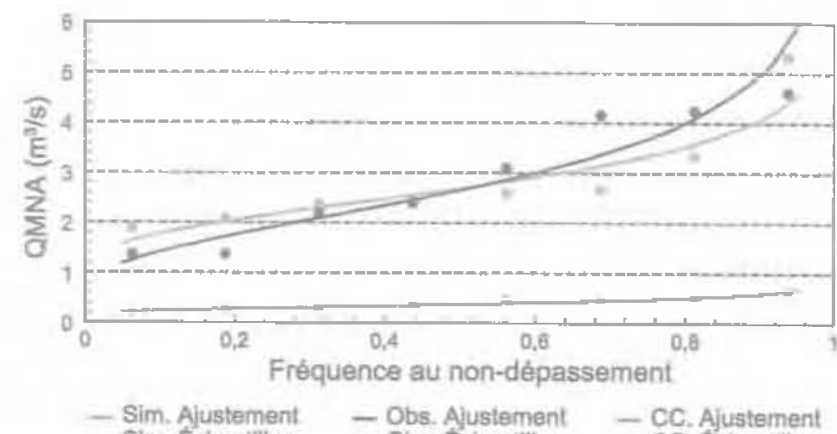

- Sim, Echantillon - Obs. Echantillon . C. Echantillon

ค6. Fonction de répartition des OMNA de la Drôme, temps présent et dans lhypothèse d'un doublement de $\mathrm{CO}_{2}$.

Repartition function of OMNA (annual minininum of monthly discharge) for the Drobme River : present time and at 2050 term with an hypothesis of $\mathrm{CO}_{2}$ doubling.

La figure 8 permet de mesurer les capacités du modèle à simuler les débits du temps présent. Les points représentent les échantillons et les courbes les 
ajustements réalisés à partir de ces échantillons. I] semblerait donc que le changement climatique entraîne une violente réduction du débit caractéristique d'étiage QMNA5. Le même résultat se retrouve pour tous les affluents du Phône étudiés.

\section{Conclusion}

Au contraire de ce qu'indiquait le regard sur le passé et la recherche de ruptures ou de tendances dans les séries hydrométéorologiques observées, une étude du futur at travers de modèles couplés hydrologie/changement climatique apporte des résultats d'une grande clarté au moins sur les étiages. Il existe donc une apparente contradiction entre les deux parties de cette étude :

1) pas de conclusion très nette par une approche de détection de tendance sur les séries hydrométéorologiques observées dans les quarante derniêres années ;

2) très fort impact du changement climatique sur be régime des rivières, notamment sur leurs étiages

Cette contradiction s'explique probablement en partie par la difficulté méthodologique associée à la détection de tendances. En effet, les signaux hydrologiques sont extremement variables par nature ; les tendances éventuelles peuvent concerner des évolutions plus complexes que celles d'une moyenne ou d'une variance et nécessiter de longues séries pour apparaître. Par ailleurs nous ne nous sommes probablement pas suffisamment écartés du fonctionnement climatique des derniers siècles, pour observer les prémices d'un glissement vers un autre fonctionnement. Pourtant, à l'aide d'une approche régionale, des tendances réelles apparaissent: dans les Alpes, augmentation des débits d'étiage, précocité de la fonte et augmentation đu volume généré par la fonte des glaciers; diminution des étiages d'été dans les Pyrénées ; légère augmentation des crues dans le Nord de la France. Ces évolutions doivent être surveillées, et des travaux complémentaires sont nécessaires avant de pouvoir les relier formellement aux évolutions climatiques attendues.

Les modèles appliqués au futur sont en revanche très affirmatifs sur l'évolution des étiages à attendre dans un avenir relativement proche : au niveau de la ressource en eau (et probablement de l'assèchement des sols), les applications réalisées ici montrent qu'il faut s'ałtendre à des diminutions importantes pendant les périodes d'été, au moins au Sud de la France. En ce qui concerne les crues et les inondations, les modèles climatiques n'apportent pas de réponse précise pour le moment. Des outils robustes restent à construire pour que les sorties des modèles climatiques soient exploitables à une maille temporelle et spatiale compatible avec les besoins de I'hydrologie des crues. Des travaux sont menés en ce sens. Deux voles sont actuellement explorées : la désagrégation statistique et la désagrégation dynamique. Dans le premier cas, il est fait usage de relations empiriques entre variables locales et des variables décrites de manfère efficace par les GCMs (paŕ exemple : Boé et al., 2006). Dans le second cas, les GCMs viennent piloter des modéles climatiques régíonaux base physique pour décrire la dynamique locale du système climatique (par exemple : Christensen et Christensen, 2006). Les deux approches sont dépendantes des progrès des GCMs qui les alimentent d'une façon ou d'une autre.

En tout état de cause, les résultats actuels doivent nous alerter: les basses eaux pourraient être nettement plus sévères dans le futur. Le principe de précaution recommande également de ne pas ignorer les quelques évolutions observées régionalement dans les régions Pyrénéennes et Alpines ou dans le Nord-Est: le rôle du changement climatique, s'il ne peut être établi formellement aujourd'hui, ne peut pas être totalement écarté.

\section{REMERCIEMENTS}

Le present article est la synthèse de travaux de recherche soutenus financièrement par le Programme National de Recherche en Hydrologie (AO2002) et par le ministère de l'Écologie, du Développement et de l'Arnénagement durables dans le cadre d'appels ä propositions GICC Gestion et Inpacts du Changement ChingGque (AO1999) et Risque Décision Territoire (AO2003). M. Lang. $E$. Leblois et E. Sauquet ont rédigé ces lignes en tant que coordinateurs des projets de recherche désormais achevés. Its tiennent à remercier l'ensemble des partenaires de leur contribution à la rếussite des projets. 
Bergstrom S., Forsman A. - Development of a conceptual deterministic rainfallrunoff model. Nordic Hydrology, 6 , 1973, p. 73-92.

Boé J., Terray L., Habets F, Martin E. Statistical and dynamical downscaling scheme based on weather types and conditional resampling. J. Geophys. Res., 111, D23106, doi:10.1029/ 2005JD006889, 2006.

Champeaux J.-L., Tamburini A. - Zonage climatique de la France à partir des séries de précipitations [1971-1990] du réseau climatologique d'État. $\mathrm{La}$ Météorologic, 14, 1995, p. 44-54

Christensen J.H., Christensen O.B. - A summary of the PRUDENCE model projections of changes in European climate by the end of this century. Climatic Change, 81, 2007, p. 7-30.

Cigizoglu H.K., Bayazit M., Onoz B. Trends in the Maximum, Mean, and Low Flows of Turkish Rivers. Journal of Hydrometeorology, 6(3), 2005, p. 280290.

Douglas A., Vogel R.M., Kroll C.N. Trends in floods and low flows in the United States: impact of spatial correlation. Journal of Hydrology, 240(1-2), 2000, p. 90-105.

Ducharne A., Théry S., Vienno, P., Ledoux E., Gomez E., Déqué M. - Influence du changement climatique sur l'hydrologie du bassin de la Seine. VertigO, vol. 4 (3), 2004, (http://www.vertigo. uqam.ca/vol4no3/art3vol4no3/agnes. ducharne_et_al.html).

Groisman P.Ya., Knight R.W., Karl T.R., Easterling D.R., Sun B., Lawrimore J.H. - Contemporary changes of the hydrological cycle over the cotiguous United States: trends derived from in situ observations. Journal of Hydrometeorol., 5, 2005, p. 64-85.

Kundzewicz Z.W., Graczyk D., Maurer T., Pińskwar I., Radziejewski M., Svensson C., Szwed M. - Trend detection in river flow series: 1. Annual maximum flow. Hydrological Sciences Journal, 50(5), 2005, p. 797-809.

Lang M., Bois P., Mestre O., Niel E., Sauquet $E$. - Détection de changements éventuels dans le régime des crues. Rapport de $1^{\text {re }}$ année, convention $n^{\circ} 02 C V 036$ (INSU), 2003, $75 \mathrm{p}$.

Lang M., Renard B. - Analyse régionale sur les extrêmes hydrométriques en France : détection de changements cohérents et recherche de causalité hydrologique. Congrès SHF « Variations climatiques et hydrologie ", Lyon, 27-28 mars 2007, La Houille Blanche, à paraître.

Leblois E. et al. - Projet GICC-Rhône. Rapport final, octobre 2004, $184 \mathrm{p}$.

Lins H.F., Slack J.R. - Streamflow trends in the United States. Geophysical Research Letters, 26(2), 1999, p. 227-
230.

Pardé M. - Fleuves et rivières. Armand Colin, 1955, $224 \mathrm{p}$.

Perrin C.- Vers une amélioration d'un modèle global pluie-débit au travers d'une approche comparative. Thèse de doctorat, INP Grenoble, 2000, 530 p.

Renard B. - Détection et prise en compte d'éventuels impacts du changement cli matique sur les extrêmes hydrologiques en France. Thèse de doctorat INP Grenoble, 2006, $362 \mathrm{p}$.

Renard B., Lang M., Bois P., Dupeyrat A. Mestre O., Niel H., Gailhard J., Lau rent C., Neppel L., Sauquet E. - Évolution des extrêmes hydrométriques en France à partir de données observées. La Houille Blanche, vol. 6, 2006, p. 48 54.

Sauquet E., Leblois E., Renard B, Haond M., Jouve D. - Examen de la vulnérabilité de la ressource en eau vis-à-vis des fluctuations climatiques. Application aux étiages du bassin du Rhône. Rap port du projet Risque Décision Terri toire MEDD, août 2005, $58 \mathrm{p}$.

Zhang X., Harvey K.D., Hogg W.D.,Yuzyk T.R. - Trends in Canadian streamflow. Water Resources Research, 37(4), 2001, p. 987-998. 